\title{
PSEUDO-COMPLEMENTED MODULAR SEMILATTICES
}

\author{
WILLIAM H. CORNISH
}

(Received 15 August 1972)

Communicated by B. Mond

\section{Introduction}

Chen and Grätzer [3], [4] have had great success in describing the properties of Stone lattices by way of representing them as triples. Their triple representation has recently been generalized to distributive pseudo-complemented lattices by Katrinák [6]. By varying the approach slightly Katriňák [5] has been able to obtain a triple representation for distributive pseudo-complemented semilattices that enabled him to characterize semilattices from distributive pseudo-complemented semilattices through to Stone lattices and Brouwer lattices in an elegant unified manner.

Two questions arise from Katriňák's work. Firstly, to what extent is distributivity necessary in order to obtain a triple representation? Secondly, is there something special about pseudo-complementation that enables a semilattice to possess some type of triple representation? We answer the first question by showing that the triple representation can be achieved for a modular pseudo-complemented semilattice, provided its filter of dense elements is neutral. This last proviso is satisfied when the semilattice is either distributive or a lattice. Our approach is achieved by way of giving a negative answer to the second question.

Thus, in section 1 of this paper modular semilattices are introduced and those properties necessary to give a triple representation in the style of Katriňák [5] are studied. Many such properties are characteristic of modularity. Incidentally, modular semilattices are not new and have been studied briefly by Varlet [12] and in great detail by Rhodes [10]. In Section 2, loosely speaking we show that a modular semilattice $S$ with 1 is represented and is uniquely determined by a triple $(C, \psi, D)$, where $C$ and $D$ are modular semilattices with 1 and $\psi$ is a 1-dual homomorphism of $C$ into the lattice of filters of $D$, if and only if $S$ possesses a multiplicative closure operator $\pi$ such that each element of $S$ has the form $c \wedge d$, where $c$ is $\pi$-closed and $d$ is $\pi$-dense, and the filter of $\pi$-dense elements is neutral. Actually $\pi$ is also determined by the triple. These results are then specialized to pseudo-complemented modular semilattices in the final section. 


\section{Modular semilattices}

Throughout this paper we shall be concerned with lower semilattices. A nonempty subset $F$ of such a semilattice $S$ is called a filter if it is subsemilattice and $a \leqq b, b \in S, a \in F$ imply $b \in F$. If $S$ is directed above then the set-theoretic intersection of two filters is a filter and the set $F(S)$ of all filters on $S$ forms a lattice under set-inclusion, where the meet of filters $F_{1}$ and $F_{2}$ is $F_{1} \cap F_{2}$ and their join is $F_{1} \vee F_{2}=\left\{s \in S: s \geqq f_{1} \wedge f_{2}\right.$ for some $f_{1} \in F_{1}$ and $\left.f_{2} \in F_{2}\right\}$. The smallest filter containing $x \in S$ is denoted by $[x)$ and is given by $[x]=\{s \in S$ : $s \geqq x\}$. Such filters are called principal.

A semilattice $S$ is called modular if, for any $x, y$ and $z$ in $S$ such that $y \geqq z$ and $z \geqq x \wedge y$, there exists $x_{1} \geqq x$ satisfying $z=x_{1} \wedge y$. This concept has been introduced in an equivalent yet slightly more complicated form by Varlet [12]. Modular semilattices have also been studied recently by Rhodes in [8], [9] and [10]. Though the following characterization is known from Rhodes' work, a proof is included since it is fundamental to the rest of the paper.

Proposition 1.1. A semilattice $S$ is modular if and only if

(i) $S$ is directed above, and

(ii) the lattice $F(S)$ of filters of $S$ is a modular lattice.

Proof. Suppose that $S$ is modular. If $x, y \in S$ then $y \geqq y$ and $y \geqq x \wedge y$ imply $y=x_{1} \wedge y$ for some $x_{1} \geqq x$. Hence $x_{1}$ is an upper bound of both $x$ and $y$ and (i) holds. Due to (i) $F(S)$ is a lattice. Suppose that $J, H, K \in F(S)$ and $J \subseteq K$. Let $x \in(J \vee H) \cap K$. Then $x \geqq a \wedge b$ for suitable $a \in J$ and $b \in H$, while $x \in K$ also. As $S$ is modular $x \wedge a \geqq a \wedge b$ and $a \geqq x \wedge a$ imply $x \wedge a=a \wedge b_{1}$ for some $b_{1} \geqq b$. Then $b_{1} \in H$. As $a \in J \subseteq K, x \wedge a \in K$ so $b_{1} \in K$ since $b_{1} \geqq x \wedge a$. Hence $b_{1} \in H \cap K$ and $x \geqq a \wedge b_{1}$ implies $x \in J \vee(H \cap K)$, and it follows that $(J \vee H) \cap K \subseteq J \vee(H \cap K)$. As the reverse inequality holds in any lattice, $F(S)$ is modular.

If (i) and (ii) hold and $x, y, z \in S$ with $y \geqq z \geqq x \wedge y$ then $[y] \subseteq[z] \subseteq[x) \vee[y)$. By the modularity of $F(S)$,

$$
z \in[z)=([y) \vee[x)) \cap[z)=[y) \vee([x) \cap[z))
$$

so $z \geqq y \wedge w$, where $w \in[x)$ i.e. $w \geqq x$, proving that $S$ is modular.

Corollary 1.2. A lattice $(L, \wedge, \vee)$ is a modular lattice if and only if the semilattice $(L, \wedge)$ is modular.

Proof. The filters of the lattice $(L, \wedge, \vee)$ are one and the same thing as those of the semilattice $(L, \wedge)$.

Before continuing with the theme of the paper some amplification might be in order. A semilattice $S$ is called distributive (prime) if for each $x, y, z$ in $S$ such 
that $z \geqq x \wedge y(x \vee y$ exists in $S)$ there exist $x_{1}$ and $y_{1}$ such that $x_{1} \geqq x, y_{1} \geqq y$ and $z=x_{1} \wedge y_{1}((z \wedge x) \vee(z \wedge y)$ exists in $S$ and

$$
z \wedge(x \vee y)=(z \wedge x) \vee(z \wedge y))
$$

Distributive semilattices were introduced by Grätzer and Schmidt. Attention was given to them by Schmidt in [11]. It is widely known that Proposition 1.1. holds if "distributive" replaces "modular", see [5], [10], [11] and [12]. Prime semilattices were studied recently by Balbes [1], who showed that a distributive semilattice is prime. The converse is not true since a prime semilattice need not be directed above, for example, consider the three element semilattice which is not a lattice. Indeed, Balbes gave an example of a directed above prime semilattice which is not distributive. It is $S=\left\{0, x, y, z, a_{0}, a_{1}, a_{2}, \cdots, a_{n}, \cdots: 0<x, y, z<a_{i}\right.$ for all $i$ and $\left.a_{0}>a_{1}>a_{2}>\cdots>a_{n}>\right\}$. We remark that Balbes' example furnishes us with an example of a modular semilattice which is not distributive. It is scarcely worth remarking that a distributive semilattice is modular and that a finite modular semilattice is a lattice. This last assertion follows from part (i) of Proposition 1.1. Rhodes' paper [10] contains a detailed treatment of modular and distributive semilattices. The rest of the results of this section were motivated by the triple construction and are independent of Rhodes' work.

Proposition 1.3. Let $F_{1}$ and $F_{2}$ be filters in a modular semilattice $S$. If $F_{1} \vee F_{2}$ and $F_{1} \cap F_{2}$ are principal then $F_{1}$ and $F_{2}$ are principal.

Proof. Let $F_{1} \vee F_{2}=[a)$ and $F_{1} \cap F_{2}=[b)$ for suitable $a, b \in S$. Then $a \geqq a_{1} \wedge a_{2}$ for some $a_{1} \in F_{1}$ and $a_{2} \in F_{2}$. But $F_{1}, F_{2} \subseteq[a)$, so $a=a_{1} \wedge a_{2}$. Take any $x$ in $F_{1}$, then since $a_{1} \wedge a_{2}=a \leqq x \wedge a_{1} \leqq a_{1}$ by modularity there is $a_{2}^{\prime} \geqq a_{2}$ such that $x \wedge a_{1}=a_{1} \wedge a_{2}^{\prime}$. Also $a_{2}^{\prime} \geqq x \wedge a_{1} \in F_{1}$ and $a_{2}^{\prime} \in F_{1} \cap F_{2}$ and $a_{2}^{\prime} \geqq b$. Therefore $x \geqq x \wedge a_{1}=a_{1} \wedge a_{2}^{\prime} \geqq a_{1} \wedge b$. This is for all $x$ in $F_{1}$, so that $F_{1}=\left[a_{1} \wedge b\right)$. A similar argument shows that $F_{2}=\left[a_{2} \wedge b\right)$.

The next result is an immediate corollary of the two preceding propositions and [2, Theorem 8, p. 187].

COROLLARY 1.4. If $a$ and $b$ are two elements of a complete modular algebraic lattice such that $a \vee b$ and $a \wedge b$ are compact, then $a$ and $b$ are both compact.

If $F$ is a filter on semilattice $S$ then $\Phi(F)$ denotes the semilat tice congruence defined by $x \equiv y[\Phi(F)](x, y \in S)$ iff $x \wedge t=y \wedge t$ for some $t \in F$. For $b \in S, \Phi_{b}$ denotes the congruence: $x \equiv y\left(\Phi_{b}\right)$ iff $x \wedge b=y \wedge b$. Of course, $\Phi_{b}=\Phi([b))$.

It is worth mentioning explicitly that $x \equiv y(\Phi(F))$ if and only if $F \vee[x)$ $=F \vee[y)$ holds in $F(S)$.

If $x, y \in S$ and $t \in F$ are such that $x \wedge y=x \wedge t$ then $[x] \Phi(F) \leqq[y] \Phi[F)$ in the factor semilattice $S / \Phi(F)$. The converse is characteristic of modularity. 
Proposition 1.5. Let $x$ and $y$ be arbitrary elements of semilattice $S$. Then, for any filter $F,[x] \Phi(F) \leqq[y] \Phi(F)$ in $S / \Phi(F)$ implies $x \wedge y=x \wedge t$ for some $t \in F$, if and only if $S$ is modular.

Proof. Suppose that $S$ is modular. If $[x] \Phi(F) \leqq[y] \Phi(F)$ holds then $x \wedge y$ $\equiv x(\Phi(F))$ so that $x \wedge y \wedge w=x \wedge w$ for some $w \in F$. Then $x \geqq x \wedge y \geqq x \wedge w$ imply, by virtue of modularity, that $x \wedge y=x \wedge t$ for some $t \geqq w$, and so $t \in F$.

Suppose that $x, y, z \in S$ and $x \wedge y \leqq z \leqq x$ and that the order in the factor semilattice of $S$ modulo an arbitrary filter $F$ is determined as announced. Choose $F=[y)$. Since $z \wedge y=x \wedge y, z \equiv x(\Phi(F))$ so that $[x] \Phi(F) \leqq[z] \Phi(F)$. Hence there is $t \in F$, i.e. $t \geqq y$, such that $x \wedge t=x \wedge z=z$, proving that $S$ is modular.

Rhodes [10] has observed that a homomorphic image of a distributive semilattice may fail to be even modular. This should be compared with the following special case.

Proposition 1.6. If $F$ is a filter on a modular (resp. distributive semilattice) $S$ then $S / \Phi(F)$ is modular (distributive).

Proof. The distirbutive case has been established by Schmidt [11, Hilfssatz 4.2]. See $[5,3.1]$ also.

Suppose that $S$ is modular. For $x \in S$, let $\bar{x}$ denote the $\Phi(F)$-class of $x$. Suppose that $a, b, c \in S$ and $\bar{a} \wedge \bar{b} \leqq \bar{c} \leqq \bar{b}$. As $S$ is modular Proposition 1.5 implies $a \wedge b \wedge c=a \wedge b \wedge w$ and $b \wedge c=c \wedge u$ for some $w, u \in F$. Hence $a \wedge c \wedge u=a \wedge b \wedge w$ so that $c \wedge u \geqq(b \wedge u) \wedge(a \wedge w)$ and $b \wedge u \geqq c \wedge u$. Since $S$ is modular $c \wedge u=(b \wedge u) \wedge x$ for some $x \geqq a \wedge w$. Then $c \equiv c \wedge u$ $\equiv(b \wedge u) \wedge x \equiv b \wedge x(\Phi(F))$ and $x \wedge a \equiv x \wedge a \wedge w \equiv a \wedge w \equiv a(\Phi(F))$. Thus $\bar{c}=\bar{b} \wedge \bar{x}$ and $\bar{x} \geqq \bar{a}$.

Suppose that $S$ is a semilattice, $C$ is a subsemilattice and $D$ is a filter on $S$. For $c \in C$, put $c \psi=\{d \in D: d \geqq c\}=D \cap[c)$. Of course $c \psi$ is a filter on both $D$ and $S . \Theta(c \psi)$ and $\Theta_{c}$ denote the restrictions of $\Phi(c \psi)$ and $\Phi_{c}$, respectively, to $D . \Phi(c \psi) \leqq \Phi_{c}$ so that $\Theta(c \psi) \leqq \Theta_{c}$. The next proposition describes when $\Theta(c \psi)$ and $\Theta_{c}$ have the same restriction on $D$.

Proposition 1.7. For a semilattice $S$, the following are equivalent:

(i) $S$ is modular,

(ii) $\Theta(a \psi)=\Theta_{a}$ for any $a \in S$ and any filter $D$,

(iii) $\Theta(a \psi)=\Theta_{a}$ for any $a \in S$ and any principal filter $D$.

Proof. (i) $\Rightarrow$ (ii). Suppose that $S$ is modular. It suffices to prove that $\Theta_{a}$ $\leqq \Theta(a \psi)$. If $x \equiv y\left(\Theta_{a}\right),(x, y \in D)$, then $x \wedge a=y \wedge a$ so

$$
[x) \vee[a)=[y) \vee[a) \text { in } F(S) .
$$

As $[x),[y] \subseteq D$, Proposition 1.1 implies 


$$
\begin{aligned}
{[x) \vee a \psi=[x) \vee([a) \cap D) } & =([x) \vee[a)) \cap D=([y) \vee[a)) \cap D \\
& =[y) \vee([a) \cap D)=[y) \vee a \psi .
\end{aligned}
$$

Hence $x \equiv y(\Phi(a \psi))$ so $x \equiv y \Theta(a \psi)$.

(ii) $\Rightarrow$ (iii) is trivial.

(iii) $\Rightarrow$ (i). Let $x, y, z \in S$ be such that $y \geqq z \geqq x \wedge y$. Put $D=[z)$ and $a=x$ and apply (iii). Since $z \wedge x=y \wedge x, z \equiv y\left(\Theta_{a}\right)$ so $z \equiv y(\Theta(a \psi))$. That is $z \wedge w$ $=y \wedge w$ for some $w \in D=[z)$ such that $w \geqq a=x$. Then $z=z \wedge w=y \wedge w$ and $w \geqq x$, so that (i) holds.

$\psi: C \rightarrow F(D)$, defined by $\psi: c \rightarrow c \psi$, is an order reversing map. In general it may not be a dual homomorphism, i.e. $\left(c \wedge c_{1}\right) \psi=c \psi \vee c_{1} \psi$ for each $c, c_{1} \in C$. (Note that the join of the filters $c \psi$ and $c_{1} \psi_{1}$ is the same in $F(D)$ as it is in $F(S)$ so that there is no ambiguity if it is simply denoted by $\left.c \psi \vee c_{1} \psi\right)$. To study this phenomenon we must recall the concept of a neutral element. An element $n$ of a lattice $L$ is called neutral if the sublattice generated by $n$ and any two other elements $x$ and $y$ of $L$ is distributive. When $L$ is modular it is well-known, see $[2$, Theorem 12, p. 37] that any "distributive equation" linking $n, x$ and $y$,

$$
\text { e.g. }(x \vee y) \wedge n=(x \wedge n) \vee(y \wedge n) \text {, }
$$

implies that all other distributive equations between $n, x$ and $y$ hold. Thus the neutral elements of modular lattices can be described by any of the various distributive equalities. A neutral filter $F$ of a directed semilattice $S$ is a filter which is a neutral element of the lattice $F(S)$.

Proposition 1.8. Let $S$ be a modular semilattice with largest element $1, C$ a sublattice and $D$ a filter on $S$. Suppose that $S, C$ and $D$ are interrelated by the condition:

for all $s \in S$, there exist $c \in C$ and $d \in D$ such that $s=c \wedge d$. Then the following are equivalent:

(i) $D$ is a neutral filter,

(ii) for any $a, b \in C,(a \wedge b) \psi=a \psi \vee b \psi$,

(iii) for any $a, b \in C, \Theta_{a \wedge b}=\Theta_{a} \vee \Theta_{b}$ holds in the lattice of congruences on $D$.

PrOOF. (i) $\Rightarrow$ (ii) is trivial in view of our preceding remarks.

(ii) $\Rightarrow$ (i). (ii) implies that

$$
([a) \vee[b)) \cap D=([a) \cap D) \vee([b) \cap D)
$$

for any $a, b \in C$.

Now let $x, y \in S$ with $x=a \wedge d$ and $y=b \wedge e$ for suitable $a, b \in C$ and $d, e \in D$. Then

$$
[x) \cap D=([a) \vee[d)) \cap D=([a) \cap D) \vee[d)
$$


since $d \in D$ and $S$ is modular. Similarly, $[y) \cap D=([b) \cap D) \vee[e)$. Thus

$$
\begin{aligned}
([x) \cap D) \vee([y) \cap D) & =([a) \cap D) \vee([b) \cap D) \vee[d) \vee[e) \\
=([a \wedge b) \cap D) \vee[d \wedge e) & =([a \wedge b) \vee[d \wedge e)) \cap D=[a \wedge b \wedge d \wedge e) \cap D \\
& =[x \wedge y) \cap D=([x) \vee[y]) \cap D,
\end{aligned}
$$

using (ii) and the modularity of $S$.

Now let $I$ and $J$ be arbitrary filters on $S$. To prove that $D$ is neutral it is sufficient to show that $(I \vee J) \cap D=(I \cap D) \vee(J \cap D)$ since $S$ is modular. For this we need only prove $(I \vee J) \cap D \subseteq(I \cap D) \vee(J \cap D)$ since the reverse inequality always holds. Thus, let $w \in(I \vee J) \cap D$, so $w \in D$ and $w \geqq x \wedge y$ for some $x \in I$ and $y \in J$. Then $w \in([x) \vee[y)) \cap D=([x) \cap D) \vee([y) \cap D) \subseteq(I \cap D) \vee(J \cap D)$.

(ii) $\Rightarrow$ (iii). Since $\Theta(a \psi)$ is the congruence on $D$ induced by the filter $a \psi$ on $D, \Theta(a \psi) \vee \Theta(b \psi)=\Theta(a \psi \vee b \psi)$. Thus, by (ii), $\Theta(a \psi) \vee \Theta(b \psi)=\Theta((a \wedge b) \psi)$. $S$ is modular so Proposition 1.7 implies $\Theta_{a} \vee \Theta_{b}=\Theta_{a \wedge b}$.

(iii) $\Rightarrow$ (ii). Using (iii) and Proposition 1.7, $\Theta((a \wedge b) \psi)=\Theta(a \psi) \vee \Theta(b \psi)$ $=\Theta(a \psi \vee b \psi)$. As $x \psi$ is order-reversing, $(a \wedge b) \psi \geqq a \psi \vee b \psi$. Now if $x \in$ $(a \wedge b) \psi$ then $x \equiv 1(\Theta((a \wedge b) \psi)=\Theta(a \psi \vee b \psi))$. Hence $x \in a \psi \vee b \psi$. (ii) follows.

\section{Neutral $p$-closure operators}

PART A. Introduction.

Let $S$ be a semilattice with largest element 1 . Recall that a map $\pi: S \rightarrow S$ is called a closure operator if (i) $(x \pi) \pi=x \pi$, (ii) $x \leqq y$ implies $x \pi \leqq y \pi$, and (iii) $x \leqq x \pi$ hold for all $x, y \in S$.

$C_{\pi}(S)=\{x \in S: x=x \pi\}$ and $D_{\pi}(S)=\{x \in S: x \pi=1\}$ are respectively called the set of $\pi$-closed elements and $\pi$-dense elements of $S$.

Closure operator $\pi$ is called normalized if $S$ has a smallest element 0 and 0 is $\pi$-closed.

Closure operator $\pi$ is called multiplicative if (iv): $(x \wedge y) \pi=x \pi \wedge y \pi$ for all $x, y$ in $S$. If $\pi$ is multiplicative then $C_{\pi}(S)$ is a subsemilattice of $S$ and $D_{\pi}(S)$ is a filter on $S$.

A p-closure operator $\pi$ on $S$ is a multiplicative closure operator such that $(v)$ : for each $s \in S$, there exist $c \in C_{\pi}(S)$ and $d \in D_{\pi}(S)$ such that $s=c \wedge d$. (v) is equivalent to $(v)^{\prime}$; for each $s \in S$ there exists $d \in D_{\pi}(S)$ such that $s=s \pi \wedge d$.

A multiplicative closure operator $\pi$ is called neutral if (vi): the filter $D_{\pi}(S)$ of $\pi$-dense elements is a neutral filter.

Proposition 2.1. Let $\pi$ be a multiplicative closure operator on semilattice $S$ with 1. Then the map $\alpha: S / \Phi\left(D_{\pi}(S)\right) \rightarrow C_{\pi}(S)$, defined by $\left([s] \Phi\left(D_{\pi}(S)\right)\right) \alpha=s \pi$, is an isomorphism if $\pi$ is a p-closure operator. 
Conversely, if $\alpha$ is an isomorphism and $S$ is modular then $\pi$ is a p-closure operator.

Proof. Suppose that $\pi$ is a $p$-closure. Then $\alpha$ is well defined because $\pi$ is multiplicative and is a homomorphism for the same reason. $\alpha$ is clearly onto and it is one-to-one since $\pi$ satisfies (v)'.

Now suppose that $S$ is modular and $\alpha$ is an isomorphism. Since $\left([s] \Phi\left(D_{\pi}(S)\right)\right) \alpha=s \pi=\left([s \pi] \Phi\left(D_{\pi}(S)\right)\right) \alpha, s \wedge d=s \pi \wedge d$ for some $d \in D_{\pi}(S)$. Hence $s \pi \geqq s \geqq s \pi \wedge d$ so that $s=s \pi \wedge d_{1}$ for some $d_{1} \geqq d$, so that $d_{1} \in D_{\pi}(S)$. Thus $\pi$ is a $p$-closure.

COROLlaRY 2.2. If $\pi$ is a p-closure operator on a modular (distributive) semilattice $S$ then $D_{\pi}(S)$ and $C_{\pi}(S)$ are both modular (distributive).

Proof. $D_{\pi}(S)$ is modular (distributive) since any filter of a modular (distributive) semilattice is clearly itself a modular (distributive) semilattice. The rest follows from Propositions 1.6 and 2.1.

PART B. The associated triple and uniqueness theorem.

If $S$ is a lower semilattice with 1 and $T$ is an upper semilattice with 0 then $\psi: S \rightarrow T$ is called a 1-dual homomorphism if $1 \psi=0$ and $(x \wedge y) \psi=x \psi \vee y \psi$ for each $x$ and $y$ in $S$. If $S$ and $T$ also have a smallest element 0 and largest element 1 , respectively, then a 1-dual homomorphism $\psi$ is called a $(0,1)$-dual homomorphism if $0 \psi=1$.

If $S$ is a modular semilattice with 1 (resp. 0 and 1 ) possessing a $p$-closure operator $\pi$ (normalized $p$-closure operator $\pi$ ) then

$$
\psi_{\pi}^{s}: C_{\pi}(S) \rightarrow F\left(D_{\pi}(S)\right), \text { defined by }
$$

$c \psi_{\pi}^{s}=\left\{d \in D_{\pi}(S): d \geqq c\right\}$ for each $c \in C$, is a 1-dual homomorphism $((0,1)$-dual homomorphism) if and only if $\pi$ is a neutral closure operator. This is because of Proposition 1.8. In this case $\psi_{\pi}^{s}$ is called the structure homomorphism associated with $S$ and $\pi$.

$\left(C_{\pi}(S), \psi_{\pi}^{s}, D_{\pi}(S)\right)$ is called the triple associated with the modular semilattice $S$ and neutral p-closure operator $\pi$.

$S$ and $\pi$ can be recovered from this triple. Indeed, if $S_{1}=\left\{c,[d] \Theta\left(c \psi_{\pi}^{s}\right)\right)$ : $\left.c \in C_{\pi}(S), d \in D_{\pi}(S)\right\}$ then $\mu: S \rightarrow S_{1}$, given by $s \mu=\left(s \pi,[d] \Theta\left((s \pi) \psi_{\pi}^{s}\right)\right.$, where $d \in D_{\pi}(S)$ is such that $s=s \pi \wedge d$, is a bijection. For, as $S$ is modular, Proposition 1.7 implies that $\Theta\left((s \pi) \psi_{\pi}^{s}\right)=\Theta_{s \pi}$ and it readily follows that $\mu$ is both welldefined and one-to-one. If $c \in C_{\pi}(S)$ and $d \in D_{\pi}(S)$ then $(c \wedge d) \mu=\left(c,[d] \Theta\left(c \psi_{\pi}^{s}\right)\right)$ so that $\mu$ is onto.

Thus we can introduce a semilattice order on $S_{1}$ and a neutral $p$-closure operator $\pi_{1}$ on $S_{1}$ such that $\mu$ becomes an isomorphism and for each $x \in S$, $(x \pi) \mu=(x \mu) \pi_{1}$. In fact, if $x, y \in S$ and $x \mu=\left(a,[d] \Theta\left(a \psi_{\pi}^{s}\right)\right), y \pi=\left(b,[e] \Theta\left(b \psi_{\pi}^{s}\right)\right.$ 
then we define the infimum in $S_{1}$ by $x \mu \wedge y \mu=\left(a \wedge b,[d \wedge e] \Theta\left((a \wedge b) \psi_{\pi}^{s}\right)\right.$ so that $S_{1}$ becomes a semilattice and it is clear that $(x \wedge y) \mu=x \mu \wedge y \mu . \pi_{1}: S_{1} \rightarrow S_{1}$ is defined by $(x \mu) \pi_{1}=\left(a,[1] \Theta\left(a \psi_{\pi}^{s}\right)\right)$ and it is readily checked that $(x \pi) \mu=$ $(x \mu) \pi_{1}$ so that $\pi_{1}$ becomes a neutral $p$-closure operator on $S_{1}$. All this can be summarized by the uniqueness theorem.

THEOREM 2.3. A modular semilattice $S$ with 1 (resp. 0 and 1) and a neutral $p$-closure operator $\pi$ is such that itself and the closure operator are determined up to a closure-isomorphism by the triple $\left(C_{\pi}(S), \psi_{\pi}^{s}, D_{\pi}(S)\right)$.

Corollary 2.2 gives the required information on the modularity or distributivity of $C_{\pi}(S)$ and $D_{\pi}(S)$.

By a closure-isomorphism $\psi: S \rightarrow T$, where $S$ and $T$ are two semilattices with closure operators $\pi$ and $\rho$ respectively, we mean an isomorphism such that $(s \psi) \rho=(s \pi) \psi$ for each $s \in S$.

PART C. Triples and the construction theorem.

$(C, \psi, D)$ is called a triple if $C$ and $D$ are both modular semilattices with 1 and $\psi$ is a 1 -dual homomorphism mapping $C$ into $F(D)$. It is a 0 -triple if $C$ has 0 and $\psi$ is a (0-1)-dual homomorphism. It is a distributive triple if both $C$ and $D$ are distributive.

With the triple $(C, \psi, D)$ we associate the set $S=\{(c,[d] \Phi c \psi)): c \in C, d \in D\}$. On $S$, a relation $\leqq$ is defined by (1) if $x=(a,[d] \Phi(a \psi)), y=(b,[e] \Phi((b \psi)) \in S$ then $x \leqq y \Leftrightarrow a \leqq b$ in $B$ and $[d] \Phi(a \psi) \leqq[e] \Phi(a \psi)$ in $D / \Phi(a \psi)$.

It is readily checked that $\leqq$ is a well-defined relation which is trivially reflexive and anti-symmetric. It is transitive since $\psi$ is order-reversing and hence is a partial order. A short computation shows that $S$ is a semilattice with respect to this order with the infimum given by

$$
x \wedge y=(a \wedge b,[d \wedge e] \Phi((a \wedge b) \psi)
$$

where $x$ and $y$ are given as in (1).

It has a largest element 1 given by

$$
1=(1,[1] \Phi(1 \psi)=(1,1),
$$

while if $(C, \psi, D)$ is a 0 -triple then $S$ has smallest element 0 given by

$$
0=(0,[1] \Phi(0 \psi))=(0, D) .
$$

$S$ is a modular semilattice. Suppose $x=(a,[d] \Phi(a \psi)), y=(b,[e] \Phi(b \psi))$ and $z=(c,[f] \Phi(c \psi))$ are in $S$ and $x \wedge y \leqq z \leqq y$. Then, from (1) and (2),

$$
a \wedge b \leqq c \leqq d,[d \wedge e] \Phi((a \wedge b) \psi) \leqq[f] \Phi((a \wedge b) \psi)
$$

and $[f] \Phi(c \psi) \leqq[e] \Phi(c \psi)$.

Since $C$ is modular, $c=a_{1} \wedge b$ for some $a_{1} \in C$ such that $a_{1} \geqq a$. 
Because $D$ is modular, Proposition 1.5 implies that $d \wedge e \wedge f=d \wedge e \wedge w$ for some $w \in(a \wedge b) \psi$ and $f \wedge e=f \wedge u$ for some $u \in c \psi$. But $(a \wedge b) \psi=a \psi \vee b \psi$ so $w \geqq v_{1} \wedge v_{2}$, where $v_{1} \in a \psi$ and $v_{2} \in b \psi$. Since $d \wedge(f \wedge u)=d \wedge e \wedge w$, we have

$$
f \wedge u \wedge v_{2} \geqq d \wedge f \wedge u \wedge v_{2} \geqq d \wedge f \wedge u \geqq d \wedge e \wedge v_{1} \wedge v_{2}
$$

That is,

$$
f \wedge\left(u \wedge v_{2}\right) \geqq\left(d \wedge v_{1}\right) \wedge\left(e \wedge v_{2}\right)
$$

Also,

$$
\left(f \wedge\left(u \wedge v_{2}\right)\right) \wedge\left(e \wedge v_{2}\right)=f \wedge\left(u \wedge v_{2}\right) .
$$

Put $f^{\prime}=f \wedge\left(u \wedge v_{2}\right), d^{\prime}=d \wedge v_{1}$ and $e^{\prime}=e \wedge v_{2}$. As $c \leqq b, b \psi \leqq c \psi$ so $v_{2} \in c \psi$ whence $u \wedge v_{2} \in c \psi$. Hence

$$
f^{\prime} \equiv f(\Phi(c \psi)) \quad d \equiv d^{\prime}(\Phi(a \psi))
$$

and $e^{\prime} \equiv e(\Phi(c \psi))$. Since $d^{\prime} \wedge e^{\prime} \leqq f^{\prime} \leqq e^{\prime}$, the modularity of $D$ implies $f^{\prime}=d_{1} \wedge e^{\prime}$ for some $d_{1} \geqq d^{\prime}, d_{1} \in D$.

Put $x_{1}=\left(a_{1},\left[d_{1}\right] \Phi\left(a_{1} \psi\right)\right)$. As $a \leqq a_{1}$ and

$$
[d] \Phi(a \psi)=\left[d^{\prime}\right] \Phi(a \psi) \leqq\left[d_{1}\right] \Phi(a \psi),
$$

(1) implies $x \leqq x_{1}$. From (2)

$x_{1} \wedge y=\left(a_{1} \wedge b,\left[d_{1} \wedge e\right] \Phi\left(\left(a_{1} \wedge b\right) \psi\right)\right)=\left(c,\left[d_{1} \wedge e\right] \Phi(c \psi)\right)=(c,[f] \Phi(c \psi))=z$ since $f \equiv f^{\prime} \equiv d_{1} \wedge e^{\prime}(\Phi(c \psi))$. The modularity of $S$ is established.

If $(C, \psi, D)$ is a distributive triple then $S$ is a distributive semilattice. Apart from the obvious change in notation the proof is the same as that of Katrinák [5, Korollar 5.4].

$S$ possesses a neutral p-closure operator $\pi$. If $x=(a,[d] \Phi(a \psi)) \in S$ then the map $x \rightarrow x \pi=(a,[1] \Phi(a \psi))$ is clearly a closure operator which is multiplicative in view of (2).

$$
\begin{aligned}
& C_{\pi}(S)=\{(a,[1] \Phi(a \psi)): a \in C\} \text { while } \\
& D_{\pi}(S)=\{(1,[d] \Phi(1 \psi)): d \in D\}=\{(1, d): d \in D\} .
\end{aligned}
$$

It then follows from (2) that $\pi$ is a $p$-closure.

Define $\psi_{\pi}^{s}: C_{\pi}(S) \rightarrow F\left(D_{\pi}(S)\right)$ by

$$
(a,[1] \Phi(a \psi)) \psi_{\pi}^{s}=\left\{(1, d) \in D_{n}(S):(1, d) \geqq(a,[1] \Phi(a \psi))\right\}=\{(1, d): d \in D
$$

and $d \in \psi\}$.

Since $\psi$ is a 1-dual homomorphism it follows that $\psi_{\pi}^{s}$ is a 1-dual homomorphism. Because $S$ is modular, $\pi$ is neutral by Proposition 1.8 . 
If $(C, \psi, D)$ is a 0 -triple then $\pi$ is normalized.

Clearly the map $\rho: D \rightarrow D_{\pi}(S)$ given by $d \rho=(1, d)$ is an isomorphism. Also $\sigma: C \rightarrow C_{\pi}(S)$ given by $c \sigma=(c,[d] \Phi(c \psi))$ is an isomorphism. If $F(\rho)$ denotes the extension of $\rho$ to $F(D)$ given by $(J) F(\rho)=\{d \rho: d \in J\}$ then $F(\rho)$ is an isomorphism and for each $c \in C, c \psi F(\rho)=c \sigma \psi_{\pi}^{s}$.

Summarizing so far we have the construction theorem.

THEOREM 2.4. Let $(C, \psi, D)$ be a triple. Then there exists a modular semilattice $S$ with 1 and a neutral p-closure operator $\pi$ on $S$ such that there are isomorphisms $\sigma: C \rightarrow C_{\pi}(S), \rho: D \rightarrow D_{\pi}(S)$ and if $\psi_{\pi}^{s}$ is the structure homomorphism associated with $S$ and $\pi$ then $c \psi F(\rho)=c \sigma \psi_{\pi}^{s}$ for each $c \in C$, where $F(\rho)$ is the isomorphism of $F(D)$ onto $F\left(D_{\pi}(S)\right)$ induced by $\rho$.

Moreover $\pi$ is normalized if $(C, \psi, D)$ is a 0 -triple and $S$ is distributive if $(C, \psi, D)$ is a distributive triple.

PART D. The fundamental theorem and the fill-in theorem.

Following Chen and Grätzer [3] or Katriňák [5] two triples $(C, \psi, D)$ and $\left(C_{1}, \psi_{1}, D_{1}\right)$ are isomorphic if there is a pair $(\sigma, \rho)$, where $\sigma$ is an isomorphism of $C$ onto $C_{1}$ and $\rho$ is an isomorphism of $D$ onto $D_{1}$ such that for each $c \in C$, $c \psi F(\rho)=c \sigma \psi_{1}$.

Like the above authors we easily obtain the fundamental theorem from Parts $\mathrm{B}$ and $\mathrm{C}$.

THEOREM 2.5. Two modular semilattices with neutral p-closure operators are closure-isomorphic if and only if their associated triples are isomorphic. Every triple (distributive triple, 0-triple) is isomorphic to a triple associated with a semilattice with 1 , and a neutral p-closure, whuich is modular (distributive, has a normalized closure).

In the next section of the paper we will consider the most important neutral $p$-closure, namely that arising from pseudo-complementation. However, the following fill-in theorem demonstrates the existence of other examples and the fact that a modular semilattice can possess many different neutral $p$-closure operators each having the same closed elements and the same dense elements.

THEOREM 2.6. Let $C$ and $D$ be modular (distributive) semilattices with 1 . If $C$ has more than one element there is a 1-dual homomorphism $\psi: C \rightarrow F(D)$ so that $(C, \psi, D)$ is a modular (distributive) triple. If $C$ has 0 then $\psi$ can be chosen so that $(C, \psi, D)$ is a 0-triple.

Proof. If $C$ has more than one element, choose a filter $J$ on $C$ such that $J \neq C$. Define $\psi: C \rightarrow F(D)$ by $x \psi=[1)$ if $x \in J$ and $x \psi=D$ if $x \in C \backslash J . \psi$ has the required properties. 


\section{Modular pseudo-complemented semilattices}

A semilattice $S$ is caleed pseudo-complemented if it has a smallest element 0 and for each $x$ in $S$ there is a necessarily unique element $x^{*}$ such that $x \wedge y=0$ if only and if $y \leqq x^{*}$ for each $y \in S$. The map $p: S \rightarrow S$ given by $x p=x^{* *}$ is a normalized multiplicative closure operator such that $C_{p}(S)$ is a Boolean algebra with the infimum as in $S$ and $a^{*}$ as the complement of $a \in C_{p}(S) .1=0^{*}$ is the largest element of both $S$ and $C_{p}(S)$. For details we refer to [5] and the references contained therein.

If $S$ is a modular pseudo-complemented semilattice then $p$ is a $p$-closure on $S$. For $x^{* *} \wedge x^{*}=0 \leqq x \leqq x^{* *}$ implies $x=x^{* *} \wedge d$, where $x^{*} \leqq d$. If $z \in S$ and $z \wedge d=0$ then $z \leqq x^{*}$ so $z=z \wedge d=0$, whence $d^{*}=0$ and so $d \in D_{p}(S)$.

In the general case we do not know whether $p$ is neutral. Of course, it is if $S$ is distributive. However, there is another important case.

The following lemma may be known though we cannot find an explicit proof in the literature. It is closely related to a result of Ore [7, Theorem 3, p. 622].

Lemma 3.1. Let $F$ be a filter on a lattice $L$. Then $\Phi(F)$ is a lattice congruence if and only if the equation

$$
(A \cap B) \vee F=(A \vee F) \cap(B \vee F)
$$

holds for any filters $A$ and $B$ on $L$.

Proof. Suppose that $\Phi(F)$ is a lattice congruence. Let $a, b \in L$. Then $a \equiv a \wedge t$ and $b \equiv b \wedge t(\Phi(F))$ for any $t \in F$. Hence $a \vee b \equiv(a \wedge t) \vee(b \wedge t)$ whence $(a \vee b) \wedge t^{\prime}=((a \wedge t) \vee(b \wedge t)) \wedge t^{\prime}$ for some $t^{\prime} \in F$.

To establish $([a) \cap[b)) \bigvee F=([a) \vee F) \cap([b) \bigvee F)$ it is sufficient to prove that if $x \in([a) \vee F) \cap([b) \vee F)$ then $x \in([a) \cap[b)) \vee F$. For such an element $x, x \geqq a \wedge t_{1}, b \wedge t_{2}$, where $t_{1}, t_{2} \in F$. Put $t=t_{1} \wedge t_{2} \in F$. From the first paragraph of this proof there is $t^{\prime} \in F$ such that

$$
x \geqq((a \wedge t) \vee(b \wedge t)) \wedge t^{\prime}=(a \vee b) \wedge t^{\prime} \in([a) \cap[b)) \vee F .
$$

It readily follows that $(A \cap B) \vee F=(A \vee F) \cap(B \vee F)$ holds for arbitrary filters $A$ and $B$.

Conversely, suppose that $F$ satisfies the given distributive equation in $F(L)$. If $a \equiv b(\Phi(F))$ then $[a) \vee F=[b) \vee F$. Hence, $[a \vee c) \vee F=([a) \cap[c)) \vee F$ $=([a) \vee F) \cap([c) \vee F)=([b) \vee F) \cap([c) \vee F)=[b \vee c) \vee F$ for any $c \in L$. That is, $a \vee c \equiv b \vee c(\Phi(F))$ for any $c \in L$. Thus $\Phi(L)$ has the substitution property for the join.

Proposition 3.2. If $L$ is a modular pseudo-complemented lattice then the filter of dense elements is neutral. 
Proof. It is well known that the relation $R$ defined by $x \equiv y(R) \Leftrightarrow x^{* *}=y^{* *}$ is a lattice congruence on any pseudo-complemented lattice $L$. For the semilattice $L, \Phi\left(D_{p}(L)\right) \subseteq R$. However, since $L$ is modular $p$ is a $p$-closure and it follows that $R \subseteq \Phi(D)$. Thus $\Phi\left(D_{p}(L)\right)=R$ is a lattice congruence and the preceding lemma shows that $D_{p}(L)$ is neutral.

Lemma 3.3. Let $(C, \psi, D)$ be a 0 -triple where $C$ is a Boolean algebra. Then the modular semilattice $S$ associated with this triple is a pseudo-complemented semilattice and the closure operator is a p-closure operator. $S$ is distributive if $D$ is distributive.

PROOF. If $x=(a,[d] \Phi(a \psi)) \in S$ then, from (1), (2) and (4) of Part C of Section $2, x \wedge y=0$ iff $y \leqq x^{*}=\left(a^{\prime},[1] \Phi\left(a^{\prime} \psi\right)\right)$, where $a^{\prime}$ is the complement of $a$ in $C$.

Since $C$ is distributive, $S$ is distributive when $D$ is.

It is clear that two pseudo-complemented semilattices are isomorphic if and only if they are $(p$-) closure isomorphic. Thus we obtain the following result from Lemma 3.3 and Theorem 2.5.

THEOREM 3.4. Two modular pseudo-complemented semilattices $S$ and $T$, each possessing a neutral ideal of dense elements, are isomorphic if and only if their associated triples $\left(C_{p}(S), \psi_{p}^{s}, D_{p}(S)\right)$ and $\left(C_{p}(T), \psi_{p}^{T}, D_{p}(T)\right)$ are isomorphic. Every 0-triple $(C, \psi, D)$ with $C$ a Boolean algebra is isomorphic to a triple associated with a modular pseudo-complemented semilattice $S$ with a neutral filter of dense elements and $S$ is distributive if and only if $D$ is distributive.

COROLLARY 3.5. Two modular pseudo-complemented lattices are isomorphic if and only if their associated triples are isomorphic. A modular pseudo-complemented lattice is distributive if and only if its filter of dense elements is itself a distributive lattice.

Proof. Two lattices are isomorphic if and only if they are isomorphic as semilattices. The first sentence then follows from Proposition 3.2 and Theorem 3.4. The second sentence follows fom Theorem 3.4 and the analogue of Corollary 1.2 for distributive lattice.

Katriňák [5] has been able to describe the triple associated with a distributive pseudo-complemented lattice when viewing it as a semilattice. His solution is complex; in [6] he gave a triple approach more suited to lattices. We have found the difficulties associated with either approach insurmountable when trying to pass to modular lattices. 


\section{References}

[1] R. Balbes, 'A representation theory for prime and implicative lattices', Trans. Amer. Math. Soc. 136 (1969), 261-267.

[2] G. Birkhoff, Lattice theory. (Amer. Math. Soc. Coll. Publ. Vol. 25, 3rd Ed. 1967).

[3] C. C. Chen and G. Grätzer, 'Stone lattices. I, Construction Theorems', Canad. J. Math. 21 (1969), 884-894.

[4] C. C. Chen and G. Gratzer, 'Stone lattices. II, Structure Theorems', Canad. J. Math. 21 (1969), 895-903.

[5] T. Katriňák, 'Die Kennzeichnung der distributiven pseudo-komplementären Halbverbände', J. fur reine und angew. Math. 241 (1970), 160-179.

[6] T. Katriňák, 'Über eine Konstruktion der distributiven pseudo-komplementären Verbände', (prepublication copy).

[7] O. Ore, 'Theory of equivalence relations', Duke Math. J. 9 (1942), 573-627.

[8] J. B. Rhodes, 'Modular semilattices', Abstract 672-659, Notices Amer. Math. Soc. 17 (1970), 272.

[9] J. B. Rhodes, 'A characterization of modular semilattices by their retracts', Abstract 678-A4, Notices Amer. Math. Soc. 17 (1970), 930.

[10] J. B. Rhodes, 'Modular and distributive semilattices', (prepublication copy).

[11] E. T. Schmidt, 'Zur Charakterisierung der Kongruenzverbände der Verbände', Mat. Căsop. 18 (1968), 3-20.

[12] J. C. Varlet, 'Modularity and distributivity in partially ordered groupoids', Bull. Soc. Roy, Sci. Liége, 38 (1969), 639-648.

The Flinders University of South Australia

Bedford Park, South Australia 5042 